\title{
Conflict Minerals from the Democratic Republic of the Congo- Global Tantalum Processing Plants, a Critical Part of the Tantalum Supply Chain
}

The U.S. Geological Survey (USGS) analyzes mineral and metal supply chains to identify and describe major components of mineral and metal material flows from ore extraction, through intermediate forms, to a final product. Supply chain analyses may be used (1) to identify risks to the United States associated with the supply of critical and strategic minerals and metals and (2) to provide greater supply chain transparency so that policymakers have the factbased information needed to formulate public policy. This fact sheet focuses on the post-mining/pre-consumer-product part of the tantalum supply chain. The USGS National Minerals Information Center (NMIC) has been asked by governmental and non-governmental organizations to provide information about tantalum, tin, tungsten, and gold (collectively known as "3TG minerals") processing facilities worldwide in response to U.S. legislation aimed at identifying and removing the supply chain links between the trade in these minerals and civil unrest in the Democratic Republic of the Congo and adjacent countries.
Post-beneficiation processing plants (generally called smelters and refineries) for 3TG mineral ores and concentrates were identified by company and industry association representatives as being the link in the $3 \mathrm{TG}$ mineral supply chain through which these minerals can be traced to their source of origin (mine). The determination of the source of origin is critical to the development of a complete and transparent conflict-free mineral supply chain (U.S. Government Accountability Office, 2012, p. 19). Tungsten processing plants were the subject of the first fact sheet in this series published by the USGS NMIC in August 2014 (Bermúdez-Lugo, 2014). Background information about historical conditions and multinational stakeholders' voluntary due diligence guidance for minerals from conflictaffected and high-risk areas is presented in the tungsten fact sheet. This fact sheet, the second in a series about 3TG minerals, focuses on the tantalum supply chain by listing selected processors that produced tantalum materials commercially worldwide during 2013-14. It does not provide any information regarding the sources of material processed in these facilities.

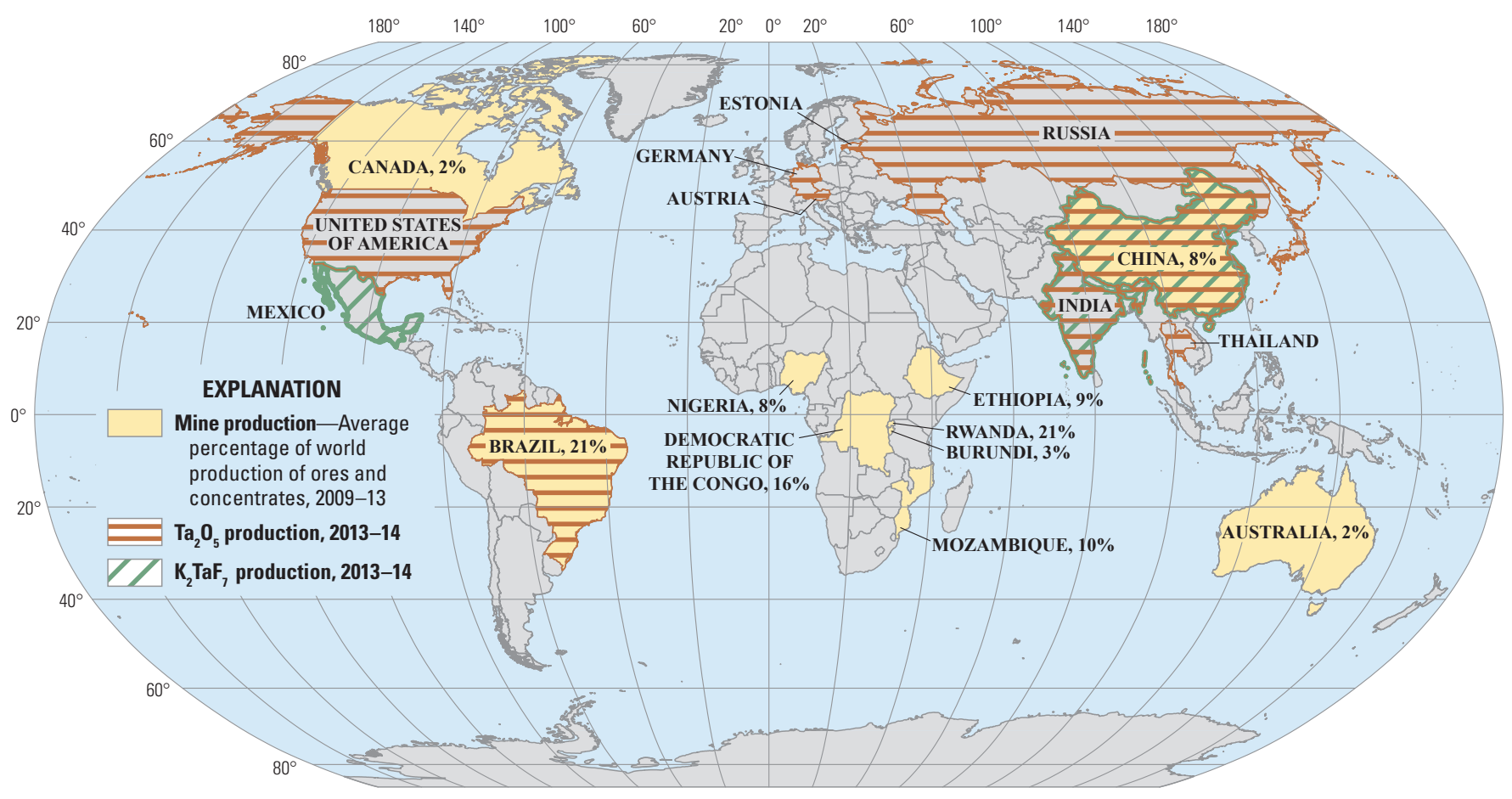

Figure 1. Global producers of tantalum industrial products in 2013-14 and percentage of 2009-13 average world tantalum mine production by country. 


\section{Background}

Tantalum does not occur naturally as pure metal; however, of the more than 70 identified tantalum-containing minerals, tantalite $\left[(\mathrm{Fe}, \mathrm{Mn})(\mathrm{Ta}, \mathrm{Nb})_{2} \mathrm{O}_{6}\right]$, microlite $\left[(\mathrm{Na}, \mathrm{Ca}) \mathrm{Ta}_{2} \mathrm{O}_{6}(\mathrm{O}, \mathrm{OH}, \mathrm{F})\right]$, and wodginite $\left[(\mathrm{Ta}, \mathrm{Nb}, \mathrm{Sn}, \mathrm{Mn}, \mathrm{Fe}) \mathrm{O}_{2}\right]$ are of greatest economic importance (Mining Journal, 2008; Roskill Information Services Ltd., 2012; Minor Metals Trade Association, 2014; Stratton and Henderson, 2014). Of these three, tantalum is derived mainly from the mineral tantalite, which is found as an accessory mineral in rare-metal granites and pegmatites enriched in lithium, cesium, and tantalum (termed the LCT family) (Schulz and Papp,

2014). Tantalum minerals also may be associated with niobium, rare-earth elements, tin, or zirconium. Tantalum is mined as a primary product and also is recovered as a byproduct from processing lithium and tin ores. Tantalum minerals are recovered by artisanal and industrial mining operations. In descending order of production, Rwanda, Democratic Republic of the Congo, and Brazil were the leading global producers of tantalum mineral concentrates in 2014. The Democratic Republic of the Congo increased tantalum production in 2013 and 2014 and replaced Brazil as the second leading producer. Figure 1 shows share of 2009-13 average world tantalum mine production, and global producers of tantalum industrial products in 2013-14 by country. The figure shows the national mine sources of tantalum, and the national processors of concentrate to the first products, $\mathrm{K}$-salt $\left(\mathrm{K}_{2} \mathrm{TaF}_{7}\right)$ and tantalum pentoxide $\left(\mathrm{Ta}_{2} \mathrm{O}_{5}\right)$.
Tantalum ore, which may be 0.02 percent to 0.04 percent $\mathrm{Ta}_{2} \mathrm{O}_{5}$, is composed of a range of different tantalum-containing minerals along with other non-tantalum-containing minerals. Owing to the low grades of tantalum ore, it is beneficiated (a physical process that does not chemically change the material) to tantalum concentrate that is 20 percent to 40 percent $\mathrm{Ta}_{2} \mathrm{O}_{5}$ equivalent (Linnen and others, 2014). The tantalum concentrate is more than 20 percent $\mathrm{Ta}_{2} \mathrm{O}_{5}$, and typically is about 30 percent $\mathrm{Ta}_{2} \mathrm{O}_{5}$.

Once the tantalum ore is recovered and concentrated, it is processed (reacted chemically at high temperature) in several different ways to extract the tantalum in a form usable by industry. Tantalum concentrate is dissolved in acid at high temperature after which the solution is reacted with chemicals that yield K-salt (also called potassium fluortantalate, potassium fluorotantalate, potassium heptafluorotantalate, or potassium tantalum fluoride) or $\mathrm{Ta}_{2} \mathrm{O}_{5}$ (also called tantalum pentoxide or tantalum oxide). Complicating the tantalum supply chain is synthetic concentrate (a byproduct of industrial processing that is a material form in which tantalum is recycled) and tantalum-containing tin slag concentrates, both of which can enter the supply chain along with tantalum concentrate produced from tantalum ore. Further processing produces tantalum metal powder and other tantalumcontaining materials. Tantalum metal is further processed into a variety of mill products (Tantalum-Niobium International Study Center, 2014).

Table 1. Selected tantalum processing plants that produced industrial tantalum products in 2013-14.

[Products produced: FeNbTa, ferroniobium-tantalum; $\mathrm{K}_{2} \mathrm{TaF}_{7}, \mathrm{~K}$-salt; $\mathrm{Ta}_{2} \mathrm{O}_{5}$, tantalum pentoxide; TaAl, aluminum alloy; TaAlloys, tantalum alloys; TaC, tantalum

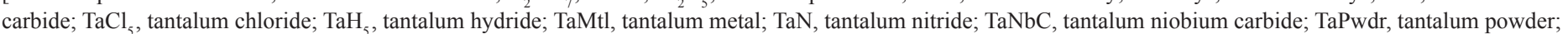
TaRecyc, tantalum recycle; $\mathrm{TaSe}_{2}$, tantalum diselenide; $\mathrm{TaSi}_{2}$, tantalum silicide. HQ, headquarters; NA, not available]

\begin{tabular}{|c|c|c|c|}
\hline Country & Product(s) produced & Location & Operator/owner \\
\hline Austria & $\mathrm{Ta}_{2} \mathrm{O}_{5}, \mathrm{TaC}$ & Multiple; Althofen, Carinthia (HQ) & Treibacher Industrie AG \\
\hline Brazil & $\mathrm{Ta}_{2} \mathrm{O}_{5}$ & São João Del Rei, Minas Gerais State & $\begin{array}{l}\text { LSM Brazil SA (formerly Companhia Industrial Flumi- } \\
\text { nense) [AMG Advanced Metallurgical Group N.V. } \\
\text { (Netherlands)] (formerly London \& Scandinavian } \\
\text { Metallurgical Co., Ltd.) }\end{array}$ \\
\hline Brazil & $\mathrm{FeNbTa}$ & São Paulo State & Mineração Taboca SA (Minsur SA) \\
\hline China & $\begin{array}{l}\text { TaPwdr, } \mathrm{Ta}_{2} \mathrm{O}_{5}, \mathrm{~K}_{2} \mathrm{TaF}_{7} \text {, } \\
\text { TaMtl }\end{array}$ & Conghua, Guangdong Province & Conghua Tantalum and Niobium Smeltery (CTNS) \\
\hline China & $\mathrm{K}_{2} \mathrm{TaF}_{7}, \mathrm{Ta}_{2} \mathrm{O}_{5}, \mathrm{TaC}$ & $\begin{array}{l}\text { Chengnan Industrial Zone, Qingyuan, } \\
\text { Guangdong Province }\end{array}$ & Fogang Jiata Metals Co., Ltd. (Jiayuan Cobalt Holdings) \\
\hline China & $\mathrm{Ta}_{2} \mathrm{O}_{5}, \mathrm{~K}_{2} \mathrm{TaF}_{7}$ & Yingde, Guangdong Province & $\begin{array}{l}\text { Guangdong Zhiyuan New Material Co., Ltd. } \\
\text { (Jiayuan Cobalt Holdings) }\end{array}$ \\
\hline China & $\mathrm{Ta}_{2} \mathrm{O}_{5}, \mathrm{~K}_{2} \mathrm{TaF}_{7}$ & NA & Hengyang King Xing Lifeng New Materials Co., Ltd. \\
\hline China & $\begin{array}{l}\mathrm{Ta}_{2} \mathrm{O}_{5}, \mathrm{~K}_{2} \mathrm{TaF}_{7}, \mathrm{TaPwdr} \\
\quad \mathrm{TaMtl}\end{array}$ & $\begin{array}{l}\text { Yaxi Industrial Development Zone, Xinhui, } \\
\text { Jiangmen, Guangdong Province }\end{array}$ & $\begin{array}{l}\text { Jiangmen Fuxiang Electro-materials Ltd. } \\
\text { (F\&X Electro-Materials Ltd.) }\end{array}$ \\
\hline China & $\mathrm{Ta}_{2} \mathrm{O}_{5}, \mathrm{~K}_{2} \mathrm{TaF}_{7}$ & Fengxin County, Jiangxi Province & Jiangxi Province Ding Hai Tantalum \& Niobium Co., Ltd. \\
\hline China & $\mathrm{Ta}_{2} \mathrm{O}_{5}, \mathrm{~K}_{2} \mathrm{TaF}_{7}, \mathrm{TaC}$ & Jiujiang, Jiangxi Province & JiuJiang JinXin Nonferrous Metals Co., Ltd. \\
\hline China & $\mathrm{Ta}_{2} \mathrm{O}_{5}$, TaMtl & Jiujiang, Jiangxi Province & $\begin{array}{l}\text { Jiujiang Tanbre Co., Ltd. (JJTC) } \\
\text { (formerly Jiujiang Tanbre smelter) } \\
\text { (Jiangxi Tungsten Group Ltd Corp. [JWYX]) }\end{array}$ \\
\hline
\end{tabular}


Table 1. Selected tantalum processing plants that produced industrial tantalum products in 2013-14. - Continued

[Products produced: FeNbTa, ferroniobium-tantalum; $\mathrm{K}_{2} \mathrm{TaF}_{7}, \mathrm{~K}$-salt; $\mathrm{Ta}_{2} \mathrm{O}_{5}$, tantalum pentoxide; TaAl, aluminum alloy; TaAlloys, tantalum alloys; TaC, tantalum carbide; $\mathrm{TaCl}_{5}$, tantalum chloride; $\mathrm{TaH}_{5}$, tantalum hydride; TaMtl, tantalum metal; TaN, tantalum nitride; TaNbC, tantalum niobium carbide; TaPwdr, tantalum powder; TaRecyc, tantalum recycle; $\mathrm{TaSe}_{2}$, tantalum diselenide; $\mathrm{TaSi}_{2}$, tantalum silicide. HQ, headquarters; NA, not available]

\begin{tabular}{|c|c|c|c|}
\hline Country & Product(s) produced & Location & Operator/owner \\
\hline China & $\begin{array}{l}\mathrm{Ta}_{2} \mathrm{O}_{5}, \mathrm{~K}_{2} \mathrm{TaF}_{7}, \mathrm{TaC} \\
\quad \text { TaMtl }\end{array}$ & Jiujiang, Jiangxi Province & $\begin{array}{l}\text { Jiujiang Zhongao Tantalum \& Niobium Co., Ltd. } \\
\text { (joint venture Jiangxi Jiujiang Yizhong Nonferrous } \\
\text { Metals Co., Ltd., and others) }\end{array}$ \\
\hline China & $\begin{array}{l}\mathrm{Ta}_{2} \mathrm{O}_{5}, \mathrm{~K}_{2} \mathrm{TaF}_{7}, \mathrm{TaC} \\
\quad \text { TaPwdr, TaMtl }\end{array}$ & $\begin{array}{l}\text { Shishi Industrial Zone, Yifeng County Indus- } \\
\text { trial Park, Fengcheng, Jiangxi Province }\end{array}$ & King-Tan Tantalum Industry, Ltd. \\
\hline China & $\begin{array}{l}\text { TaAlloys, } \mathrm{TaC}_{2} \mathrm{Ta}_{2} \mathrm{O}_{5} \\
\text { TaN, } \mathrm{TaSi}_{2}, \mathrm{TaSe}_{2}\end{array}$ & $\begin{array}{l}\text { Many (Shanghai, Suzhou in China and } \\
\text { others in England, Ireland, Philippines, } \\
\text { Singapore, Taiwan, and United States) }\end{array}$ & Materion Advanced Materials Thin Film Products \\
\hline China & $\mathrm{Ta}_{2} \mathrm{O}_{5}$, TaMtl, TaPwdr & $\begin{array}{l}\text { Jiangning Economic Development Zone, } \\
\text { Nanjing, Jiangsu Province }\end{array}$ & $\begin{array}{l}\text { Metalink International Co., Ltd. } \\
\text { (affiliates: Nanjing Metalink International Co., Ltd., } \\
\text { and Metalink Special Alloys Corp.) }\end{array}$ \\
\hline China & $\begin{array}{l}\mathrm{Ta}_{2} \mathrm{O}_{5}, \mathrm{TaCl}_{5}, \mathrm{TaNbC} \\
\text { Ta ethoxide, TaMtl, } \\
\text { TaAlloys }\end{array}$ & Shizuishan, Ningxia Province & Ningxia Orient Tantalum Industry Co., Ltd. (OTIC) \\
\hline China & $\mathrm{Ta}_{2} \mathrm{O}_{5}$ & $\begin{array}{l}\text { Buxia Cun, Rencun Town, Xinxing, } \\
\text { Yunfu, Guangdong Province }\end{array}$ & XinXing Haorong Electronic Material Co., Ltd. \\
\hline China & $\mathrm{Ta}_{2} \mathrm{O}_{5}, \mathrm{TaC}, \mathrm{TaPwdr}$ & $\begin{array}{l}\text { Lianggang Industrial Park, Yifeng County, } \\
\text { Yichun, Jiangxi Province }\end{array}$ & Yichun Jinyang Rare Metal Co., Ltd. \\
\hline China & $\begin{array}{l}\mathrm{K}_{2} \mathrm{TaF}_{7}, \mathrm{Ta}_{2} \mathrm{O}_{5} \text {, } \\
\quad \text { Ta ethoxide, TaPwdr, } \\
\text { TaMtl, TaAlloys, TaC }\end{array}$ & Zhuzhou, Hunan Province & $\begin{array}{l}\text { Zhuzhou Cemented Carbide Works Import \& Export Co. } \\
\text { (Zhuzhou Cemented Carbide Group Corporation, Ltd.) }\end{array}$ \\
\hline Estonia & $\begin{array}{l}\mathrm{Ta}_{2} \mathrm{O}_{5}, \mathrm{TaH}_{5}, \mathrm{TaMtl} \\
\quad \text { (ingots, chips) }\end{array}$ & Sillamae, Ida-Virumaa & Molycorp Silmet AS (Molycorp, Inc.) \\
\hline Germany & TaMtl, $\mathrm{Ta}_{2} \mathrm{O}_{5}, \mathrm{TaAl}$ & Freiberg, Saxony & $\begin{array}{l}\text { Freiberger NE-Metall GmbH \& Co. Produktions KG } \\
\text { (GfE Gesellschaft für Elektrometallurgie mbH, AMG } \\
\text { Advanced Metallurgical Group N.V.) }\end{array}$ \\
\hline Germany & TaMtl, $\mathrm{Ta}_{2} \mathrm{O}_{5}, \mathrm{TaAl}$ & Nuremberg, Middle Franconia & $\begin{array}{l}\text { GfE_-Metalle und Materialien GmbH } \\
\text { (GfE Gesellschaft für Elektrometallurgie mbH, } \\
\text { AMG Advanced Metallurgical Group N.V.) }\end{array}$ \\
\hline Germany & $\begin{array}{l}\text { TaPwdr, TaNbC, } \mathrm{Ta}_{2} \mathrm{O}_{5}, \\
\mathrm{TaCl}_{5}, \mathrm{TaMtl}, \mathrm{TaRecyc}\end{array}$ & $\begin{array}{l}\text { Many (Munich [HQ]; Im Schleeke, Goslar, } \\
\text { Lower Saxony; Hermsdorf, Thuringia; } \\
\text { two plants in Laufenberg, Baden-Wurt- } \\
\text { temberg in Germany; others in China, } \\
\text { Japan, Thailand, and United States) }\end{array}$ & H.C. Starck, GmbH \\
\hline Germany & $\begin{array}{l}\text { TaPwdr, TaNbC, } \mathrm{Ta}_{2} \mathrm{O}_{5}, \\
\mathrm{TaCl}_{5}, \mathrm{TaMtl}, \mathrm{TaRecyc}\end{array}$ & Hanau, Hesse & $\begin{array}{l}\text { Heraeus Materials Technology, GmbH \& Co. KG } \\
\text { (formerly W.C. Heraeus) (Heraeus Holding, GmbH) }\end{array}$ \\
\hline India & $\mathrm{K}_{2} \mathrm{TaF}_{7}, \mathrm{Ta}_{2} \mathrm{O}_{5}$ & Taloja, District Raigad, Maharashtra & Metallurgical Products India Pvt Ltd. (MPIL) \\
\hline Japan & $\mathrm{Ta}_{2} \mathrm{O}_{5}, \mathrm{TaC}, \mathrm{TaNbC}$ & Omuta, Fukuoka Prefecture & $\begin{array}{l}\text { Engineered Powders Div. Miike Rare Metal Plant } \\
\text { (Mitsui Mining and Smelting Co., Ltd.) }\end{array}$ \\
\hline Japan & TaPwdr & Aizuwakamatsu, Fukushima Prefecture & Global Advanced Metals, Pty, Ltd. \\
\hline Japan & $\begin{array}{l}\text { TaPwdr, TaNbC, } \mathrm{Ta}_{2} \mathrm{O}_{5}, \\
\mathrm{TaCl}_{5}, \mathrm{TaMtl}, \mathrm{TaRecyc}\end{array}$ & Hitachiomiya, Ibaraki Prefecture & H.C. Starck, Ltd., H.C. Starck, GmbH \\
\hline Japan & $\mathrm{Ta}_{2} \mathrm{O}_{5}$ & Kako, Hyogo Prefecture & Taki Chemicals Co., Ltd. \\
\hline Mexico & $\mathrm{K}_{2} \mathrm{TaF}_{7}$ & Matamoros, Tamaulipas & $\begin{array}{l}\text { Kemet de México, S.A. de C.V. } \\
\text { (Kemet Blue Powder Corp. [Kemet Corp.]) }\end{array}$ \\
\hline Russia & $\mathrm{Ta}_{2} \mathrm{O}_{5}$, TaMtl & Solikamsk, Perm Krai & $\begin{array}{l}\text { Solikamsk Magnesium Works OAO } \\
\text { (Solikamsk Magnesium Works [SMZ] JSC) }\end{array}$ \\
\hline United States & TaMtl, TaAlloys & $\begin{array}{l}\text { Boyertown, Berks and Montgomery } \\
\text { Counties, Pennsylvania }\end{array}$ & Global Advanced Metals \\
\hline
\end{tabular}




\section{Uses}

Tantalum has many properties that make it desirable for a wide array of applications. Tantalum can store and release energy, making it desirable to the electronics industry, which consumes more than one-half of tantalum world mine production. Tantalumbased components can be exceptionally small, and other chemical elements cannot serve as substitutes without degrading the performance of electronic devices. As a result, tantalum is used in components for items as ubiquitous as cell phones, hard drives, and hearing aids. Tantalum's high biocompatibility allows it to coat stronger, less costly substrates, such as stainless steel, for medical applications. It is used for blood vessel support stents, bone replacements, plates, and suture clips and wire. In the chemical industry, tantalum's corrosion resistance makes it useful as a lining for pipes, tanks, and vessels. The hardness of tantalum carbide makes it an ideal component in the manufacture of cutting tools, and tantalum oxide can increase the refractive index of lens glass (Schwela, 2010).

\section{Tantalum Processing Facility Data}

More than 30 tantalum processors were part of the global tantalum supply chain during 2013-14 (table 1). The USGS does not have complete data on the source materials or processing methods of these tantalum processing companies and (or) plants and therefore cannot verify whether these facilities consumed tantalum concentrates, and, if so, whether or not those concentrates were sourced from conflict-affected areas. Other forms of tantalum such as synthetic concentrate, tin slag, or intermediate products may be used as source materials. The table excludes companies and (or) plants that were known to only process recycled materials. The tantalum supply chain is complex and, often, opaque. Some companies operate plants at multiple locations within, between, or among countries. Companies usually report products that they supply to the market place; however, they may not describe which of their plants use which starting materials and (or) processes. As a result, plants reported for some multi-plant corporations may or may not consume and (or) produce all of the tantalum materials reported. Selected tantalum processing plants are also potential tantalum concentrate consumers as indicated by their offering K-salt and (or) tantalum oxide products, the usual first materials derived from tantalum concentrate, or by their reporting that they consume concentrate.

The data were compiled and evaluated by the NMIC from sources that include company reports, foreign governments, industry analysts, industry associations, inter-governmental organizations, non-governmental organizations, and trade journals. Company information, such as name, location, and products produced, were verified via company Web sites, which were the authoritative sources for company name and location. It was the intent to include all concentrate-consuming companies and plants; however, some may not have been listed. Some large companies have multiple plants, which may or may not have been described in sufficient detail to identify their location or the tantalum material that was processed. For example, information was not available on all tantalumproducing companies, and many companies that were reported to have been tantalum suppliers could not be confirmed as such. Companies changed names, were referred to imprecisely, changed ownership, or went out of business.

\section{References Cited}

Bermúdez-Lugo, Omayra, 2014, Conflict minerals from the Democratic Republic of the Congo - Global tungsten processing plants, a critical part of the tungsten supply chain: U.S. Geological Survey Fact Sheet 2014-3069, 4 p., revised August 15, 2014. Accessed October 1, 2014, at http://pubs.usgs.gov/fs/2014/3069/.

Linnen, Robert, Trueman, D.L., and Burt, Richard, 2014, Tantalum and niobium, in Gunn, Gus, ed., 2014, Critical metals handbook: British Geological Survey, p. 361-384.

Mining Journal, 2008, Tantalum (2006 and first half of 07): Mining Journal, unpaginated.

Minor Metals Trade Association, 2014, Minor metals in the periodic table-Ta-Tantalum, accessed October 1, 2014, at http://www.mmta.co.uk/metals/Ta/.

Roskill Information Services Ltd., 2012, Tantalum-Market outlook to 2016: London, United Kingdom, Roskill Information Services Ltd., 164 p.

Schulz, Klaus, and Papp, John, 2014, Niobium and tantalumIndispensable twins: U.S. Geological Survey Fact Sheet 2014-3054, 2 p. (Also available at $h t t p: / / d x . d o i$. org/10.3133/fs20143054.)

Schwela, Ulric, 2010, The state of tantalum mining: London, United Kingdom, Mining Journal Supplement, September, $11 \mathrm{p}$.

Stratton, Patrick, and Henderson, David, 2014, Tantalum market overview: Minor Metals Trade Association, accessed October 1, 2014, at http://www.mmta.co.uk/ tantalum-market-overview/.

Tantalum-Niobium International Study Center, 2014, Tantalum-Raw materials and processing, accessed October 1, 2014, at http://tanb.org/tantalum/.

U.S. Government Accountability Office (GAO), 2012, Report to Congressional committees - Conflict minerals disclosure rule-SEC's actions and stakeholder-developed initiatives: Washington, D.C., U.S. GAO, Report GAO-12-763, 45 p. (Also available at http://www.gao.gov/ assets/600/592458.pdf.)

By John F. Papp

\section{Information Contact}

Director, National Minerals Information Center

U.S. Geological Survey

12201 Sunrise Valley Drive

988 National Center

Reston, VA 20192

Email: nmicrecordsmgt@usgs.gov

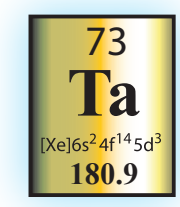

Or visit the USGS Minerals Information Web site at http://minerals.usgs.gov/minerals/ 